\title{
Time for System Redesign
}

\author{
Eva Aagaard, M.D. ${ }^{1,3}$ and Arianne Teherani, Ph.D. ${ }^{2}$
}

'University of Colorado School of Medicine, Aurora, CO, USA; ${ }^{2}$ University of California San Francisco School of Medicine, San Francisco, CA, USA; ${ }^{3}$, Aurora, CO, USA.

KEYWORDS: medical education; duty hours; transitions in care; attending physician.

$\mathrm{J}$ Gen Intern Med 30(9):1239-40

DOI: $10.1007 / \mathrm{s} 11606-015-3426-\mathrm{Z}$

(c) Society of General Internal Medicine 2015

$\mathrm{M}$ edical education and the clinical world within which it is practiced have undergone unprecedented change in the last 10 years. Duty hour reform and the shift toward outcomes-based education and assessment have had a marked impact on our educational systems. ${ }^{1}$ At the same time, many of our hospitals and clinics have undergone significant systems changes. Electronic medical records have swept the nation and, along with them, increases in physician tasks and non-face-to-face communication with patients and interprofessional colleagues. Documentation and clinical productivity pressures have increased, clinic visits have been shortened, and the pressure to reduce hospital length of stay has increased. Meanwhile, patients are seemingly getting older and sicker.

These changes to the medical education and clinical world have left internal medicine physicians, whether based in the hospital or the clinic, debating and lamenting their impact. Concerns have included apprehension about the effect of these changes on the quality of patient care, patient safety, and resident education. These often heated discussions have occurred within the walls of our institutions and in public forums, including JGIM.

While the debate continued, program directors in internal medicine were forced to move quickly. Perhaps the most common change was the transition from overnight call to shift-based systems. Designs vary from institution to institution, but most involve daytime shifts, with swing and night float shifts for admitting and cross-cover of patients. The day team is responsible for admitting new patients that come in during the day, but also for caring for their patients and those admitted overnight by the swing and night float residents. Another growing change is the shift to $\mathrm{X}+\mathrm{Y}$ schedules, wherein residents spend a fixed number of weeks on inpatient duty (generally 3-5 weeks), followed by a week or more of outpatient, largely continuity clinic care. Again, debate has raged over the clinical and educational challenges associated with these models. The impacts of these changes have been

Published online July 15, 2015 studied, but much remains unknown. What is clear is that although team continuity decreases and handoffs amongst residents increase, ${ }^{2}$ there does not appear to be any impact on patient outcomes or resident board pass rates., ${ }^{3,4}$

While no single change is to blame, there can be little doubt that physician and resident work has become progressively more challenging. Perhaps relatedly, physician stress and burnout are at staggeringly high levels. ${ }^{5}$ In this issue of JGIM, we have the opportunity to explore the impact of these many changes on our systems, learners, teachers, interprofessional colleagues, and the patients for whom we care.

In a prospective observational study spanning 12 years and two rounds of duty hour reform, Arora et al. found that after successive residency duty hours limits, hospitalized patients were more likely to report the attending physician and less likely to report the resident or intern as most involved in their hospital care. ${ }^{6}$ While there are several possible explanations for these results, they appear to confirm prior concerns by attending physicians that their workload has increased since duty hour reform. Distressingly, Arora and her team found that approximately one third of patients did not know who was most involved in their care.

In another study in this issue, we begin to see some evidence that helps explain why patients don't know who their doctors are. Using social network analysis, Pinelli and colleagues describe communication patterns surrounding a pivotal point in hospital patient care-patient discharge. ${ }^{7}$ The researchers found that discharges involve a complex network of both synchronous and asynchronous communication amongst multiple providers and team members, with interns positioned at the center of this network. Given the risks associated with transitions in care, the study raises important questions about whether it is appropriate for interns, who are amongst the most junior team members, to play this central role. Should a more experienced provider serve this function? What systems need to be put into place to ensure that these discharges are safe and effective? Can and should interprofessional teams collaborate to ensure the safety and effectiveness of these discharges?

Interprofessional collaborative care, the process during which providers work together to improve healthcare quality, is a national health policy focus as well as a growing area of medical education. Many believe it to be core to the future of high-value, patient-centered care. Soones et al. provide some insight into interprofessional collaborative care through a qualitative study that explores the factors internal medicine 
residents identified as affecting their ability to provide and learn about interprofessional team-based care, with a focus on residents' continuity clinics. ${ }^{8}$ Although residents believed teams could improve patient care and healthcare provider job satisfaction, the opportunities to experience this and learn from these experiences were hindered by the system within which they worked. Specific challenges included absences created by residents' schedules, frequent team member turnover, low staffing ratios, and a negative cultural perception that residents will do all of the work when teams break down. A recurring theme across these challenges is a lack of continuity among team members, limiting accountability and effective communication and teamwork.

Finally, studies by Patel et al. and Ryskina and colleagues investigate the role that attending providers play in the delivery of high-value and cost-conscious care by residents. ${ }^{9,10}$ Both studies validate that attendings are important role models, yet only about half model cost-conscious care. Moreover, this role-modeling does not appear to be changed by the presence of a curriculum focused on high-value, costconscious care.

So, where do we go from here? We live in a world of change. These changes are occurring in the clinical environments within which we and our learners practice, as well as the educational systems within which we teach. However, changes are occurring independently, yet simultaneously, often without regard to their interrelated impact on the broader system. It is time for us to change this. As general internists, we are among the best-positioned to deliberately examine the microand macro-systems within which we work. We span both inpatient and outpatient settings, and often provide the majority of clinical teaching in both domains. We can make the changes that need to be made to ensure we achieve our collective goals of delivering high-quality, interprofessionally collaborative, cost-effective care, while training the next generation to do the same, and yet improve upon it through innovation and system redesign.

The articles in this issue point us to some important guiding principles to consider as we move forward. First, effective communication is key-between leaders, within interprofessional teams, and across systems and hierarchies. Second, continuity supports effective communication and teamwork. As we design educational and clinical system, ensuring that we maximize continuity among learners, attendings, and interprofessional teams is critical.
Third, while many changes can occur from the bottom up, we must put time and resources into developing our faculty if we hope to create systems and learning environments that will support rather than undo the training we are providing to our learners on issues such as teamwork, interprofessional roles and responsibilities, high-value care, and clinical system redesign. Finally, we must engage learners, interprofessional team members, and attendings in redesign efforts if we hope to create systems that truly work and achieve our collective goals. The time to engage is now, and we as internists, clinicians, and educators must assume our critical role as leaders and participants in the process.

Conflict of Interest: The authors declare that they do not have a conflict of interest.

Corresponding Author: Eva Aagaard, M.D.; , Room 4102A, 13070 East 19th Avenue, Mail Stop F495, Aurora, CO 80045, USA (e-mail: Eva.Aagaard@ucdenver.edu).

\section{REFERENCES}

1. Nasca TJ, Philibert I, Brigham T, Flynn TC. The next GME accreditation system-rationale and benefits. N Engl J Med. 2012;366:1051-6.

2. Gordon M, Findley R. Educational interventions to improve handover in health care: a systematic review. Med Educ. 2011;45:1081-9.

3. Patel MS, Volpp KG, Small DS, et al. Association of the 2011 ACGME resident duty hour reforms with mortality and readmissions among hospitalized Medicare patients. JAMA. 2014;312:2364-73.

4. Rajaram R, Chung JW, Jones AT, et al. Association of the 2011 ACGME resident duty hour reform with general surgery patient outcomes and with resident examination performance. JAMA. 2014;312:2374-84.

5. Shanafelt TD, Boone S, Tan $\mathbf{L}$, et al. Burnout and satisfaction with worklife balance among US physicians relative to the general US population. Arch Intern Med. 2012;172:1377-85.

6. Arora V, Prochaska M, Farnan J, Meltzer D. Patient perceptions of whom is most involved in their care with successive duty hour limits. J Gen Intern Med. 2015 doi:10.1007/s11606-015-3239-0.

7. Pinelli V, Papp K, Gonzalo J. Interprofessional communication patterns during patient discharges: a social network analysis. J Gen Intern Med. 2015 doi:10.1007/s11606-015-3415-2.

8. Soones TN, O'Brien BC, Julian KA. Internal medicine residents' perceptions of team-based care and its educational value in the continuity clinic: a qualitative study. J Gen Intern Med. 2015 doi:10.1007/s11606-015-3228-3.

9. Patel M, Reed D, Smith C, Arora V. Role-modeling cost-conscious care-a national evaluation of perceptions of faculty at teaching hospitals in the united states. J Gen Intern Med. 2015 doi:10.1007/s11606-015-3242-5.

10. Ryskina K, Dine J, Kim E, Bishop T, Epstein A. Effect of attending practice style on generic medication prescribing by residents in the clinic setting: an observational study. J Gen Intern Med. 2015 doi:10.1007/ s11606-015-3323-5 . 